\title{
Novel Techniques for Built-Up Area Extraction from Polarimetric SAR Images
}

\author{
Debanshu Ratha, Student Member, IEEE, Paolo Gamba, Fellow, IEEE \\ Avik Bhattacharya, Senior Member, IEEE, and Alejandro C. Frery, Senior Member, IEEE
}

\begin{abstract}
Built-up area extraction from remote sensing images is important to monitor and manage urbanization and industrialization. In this work, we propose two built-up area extraction techniques based on the analysis of fully PolSAR data. Both methods exploit the geodesic distance on the unit sphere in the space of Kennaugh matrices. The first method is based on the three dominant scattering types in the scene and compares them with scattering models; if any of them matches with built-up type elementary scattering models, then the pixel is said to belong to a built-up area. The second method is based on a novel PolSAR built-up index (RBUI) composed by considering scattering mechanisms from built-up structures. The two proposed techniques are validated on two different urban scenes, one acquired at C-band by RADARSAT-2 and other at L-band by ALOS-2 SAR sensors.
\end{abstract}

Index Terms-SAR Polarimetry, urban, built-up, mapping, geodesic distance, radar built-up index (RBUI), scattering similarity, Kennaugh matrix.

\section{INTRODUCTION}

The mapping of urban areas in the context of Earth Observation (EO) has been carried out mostly by very high resolution (VHR) optical data. An in-depth review of the importance of human settlements mapping in EO is given in [1], [2].

Recent interest has arisen in using Synthetic Aperture Radar (SAR) sensors for EO as an alternative. Active SAR sensors are useful for EO applications because of their all-weather and day-night ability to acquire images. In the literature, intensity SAR data have been used for built-up area mapping [3][5]. Target descriptions provided by full-polarimetric SAR (PolSAR) data might be even more useful. Nevertheless, they are hard to employ due to the complex interactions in urban areas among the electromagnetic waves, the targets, and their surroundings. Therefore, the main avenues for extracting builtup area information from PolSAR imagery range from using decomposition theorems to feeding the data into deep learning architectures.

D. Ratha and A. Bhattacharya are with Centre of Studies in Resources Engineering, Indian Institute of Technology Bombay, India - 400076. (e-mail: debanshu.ratha@gmail.com, avikb@csre.iitb.ac.in)

P. Gamba is with the Department of Electronic, Biomedical and Computer Engineering, University of Pavia, Pavia 27100, Italy. (email: paolo.gamba@unipv.it)

Alejandro C. Frery is with Laboratório de Computação Científica e Análise Numérica - LaCCAN, Universidade Federal de Alagoas, Maceió, Brazil. (email: acfrery@laccan.ufal.br)

The first author will like to thank the Council of Scientific and Industrial Research (CSIR), Government of India for supporting his doctoral studies. Special thanks to Dr. Shaunak De for providing the reference map of the two data sets. The first author would like to thank Dr. Armando Marino for some useful discussions.
Specifically, in 2013, Kajimoto et al. [6] proposed an urban area extraction method by a two-step classification technique. Azmedroub et al. [7] used a model-based scattering decomposition powers in one method and the coefficients of scattering covariance matrix in the circular polarization basis in another method. Duan et al. [8] introduced a correlation coefficient to characterize scattering mechanisms of urban targets affected by azimuthal orientation. These methods are solely based on the discrimination of orthogonal and oriented structures about the radar line of sight (LOS) within the built-up area. Xiang et al. [9] combined reflection asymmetry with azimuth nonstationarity to improve the detection accuracy of human-made targets. In another study, Xiang et al. [10] used decision level fusion of built-up area information from a multiple-component model-based scattering decomposition method and a new coherence coefficient from a sub-aperture decomposition. $\mathrm{Wu}$ et al. [11] proposed a methodology involving nonzero-mean statistical models to differentiate human-made and natural targets. Even though the reflection symmetric property has excellent potential for built-up area target discrimination, there is a trade-off between the required number of sub-aperture for the detection of targets [9] in the built-up area. Finally, De et al. [12] used a novel data augmentation strategy in conjunction with a stacked auto-encoder architecture to improve the generalization capabilities of a neural network for enhanced urban classification.

From the processing point of view, these state-of-the-art methods span from decision trees to mixture models to deep learning respectively. In general, they require either long processing steps for backscatter analysis or manual setting of the hyper-parameters. This work aims at completing this scenario with a technique that is direct and intuitive, as well as easy to implement. Another objective is introducing a novel pixel-based built-up index in PolSAR based on exploiting scattering mechanisms.

In [13] the cross-polarized component is found to be useful to distinguish between forests and oriented urban buildings. Ref. [14] considers the effect of the double Brewster angle on dihedrals which strongly affects the $V V$ amplitude. Finally [15] studies the backscatter response from rotated dihedrals for which the signature is often very confusing. Bearing this in mind, in this study we propose a scattering similarity-based approach using the observed data and specific elementary scattering models. Structures within built-up areas show a high degree of similarity with narrow dihedral and dihedral models [16], while the cross-polarized component exists only with the helix models. Additionally, a new radar 
built-up index (RBUI) is proposed and validated in two test locations with data acquired by two different PolSAR sensors.

\section{Methodology}

In PolSAR, the $2 \times 2$ complex scattering matrix $\mathbf{S}$ encompasses complete polarimetric information about backscattering data from targets:

$$
\mathbf{S}=\left[\begin{array}{cc}
S_{H H} & S_{H V} \\
S_{V H} & S_{V V}
\end{array}\right]
$$

where the subscript $H$ and $V$ denote horizontal and vertical polarizations respectively. Following the reciprocity theorem in the case of a monostatic radar, $S_{H V}=S_{V H}$.

A suitable representation of PolSAR data in terms of power is given by the $4 \times 4$ real Kennaugh matrix $\mathbf{K}$ defined as [17]:

$$
\mathbf{K}=\frac{1}{2} A^{*}\left(S \otimes S^{*}\right) A^{H}, \quad A=\left[\begin{array}{cccc}
1 & 0 & 0 & 1 \\
1 & 0 & 0 & -1 \\
0 & 1 & 1 & 0 \\
0 & j & -j & 0
\end{array}\right]
$$

where $\otimes$ is the Kronecker product, and $j=\sqrt{-1}$.

Alternatively, the Kennaugh matrix for the incoherent target scattering can be written in terms of the elements of the coherency matrix $\mathbf{T}$ as:

$$
\mathbf{K}=\left[\begin{array}{cccc}
\frac{T_{11}+T_{22}+T_{33}}{2} & \Re\left(T_{12}\right) & \Re\left(T_{13}\right) & \Im\left(T_{23}\right) \\
\Re\left(T_{12}\right) & \frac{T_{11}+T_{22}-T_{33}}{2} & \Re\left(T_{23}\right) & \Im\left(T_{13}\right) \\
\Re\left(T_{13}\right) & \Re\left(T_{23}\right) & \frac{T_{11}-T_{22}+T_{33}}{2} & -\Im\left(T_{12}\right) \\
\Im\left(T_{23}\right) & \Im\left(T_{13}\right) & -\Im\left(T_{12}\right) & \frac{-T_{11}+T_{22}+T_{33}}{2}
\end{array}\right]
$$

where $\Re$ and $\Im$ denote the real and imaginary parts of a complex number.

There are a number of elementary scattering models: dihedral $(d)$, narrow dihedral $(n d)$, trihedral $(t)$, cylinder $(c)$, dipole $(d p)$, quarter-wave devices $( \pm 1 / 4)$, left helix $(l h)$, and

\begin{tabular}{|c|c|c|c|c|}
\hline Target & Row 1 & Row 2 & Row 3 & Row 4 \\
\hline $\mathbf{K}_{d}$ & 1000 & 0100 & $00-10$ & 000001 \\
\hline $\mathbf{K}_{n d}$ & $\begin{array}{lllll}5 / 8 & 3 / 8 & 0 & 0\end{array}$ & $3 / 85 / 800$ & $\begin{array}{llll}0 & 0 & -1 / 2 & 0\end{array}$ & $\begin{array}{llll}0 & 0 & 0 & 1 / 2\end{array}$ \\
\hline $\mathbf{K}_{t}$ & 1000 & 0100 & $\begin{array}{llll}0 & 0 & 1 & 0\end{array}$ & $\begin{array}{lllll}0 & 0 & 0 & -1\end{array}$ \\
\hline $\mathbf{K}_{c}$ & $5 / 8 \quad 3 / 800$ & $3 / 85 / 800$ & $\begin{array}{llll}0 & 0 & 1 / 2 & 0\end{array}$ & $\begin{array}{llll}0 & 0 & 0 & -1 / 2\end{array}$ \\
\hline $\mathbf{K}_{d p}$ & $1-100$ & -11000 & $\begin{array}{llll}0 & 0 & 0 & 0\end{array}$ & 00000 \\
\hline $\mathbf{K}_{+1 / 4}$ & 1000 & $\begin{array}{llll}0 & 1 & 0 & 0\end{array}$ & $\begin{array}{lllll}0 & 0 & 0 & 1\end{array}$ & $\begin{array}{llll}0 & 0 & 1 & 0\end{array}$ \\
\hline $\mathbf{K}_{-1 / 4}$ & 1000 & 0100 & $\begin{array}{lllll}0 & 0 & 0 & -1\end{array}$ & $\begin{array}{lllllll}0 & 0 & -1 & 0\end{array}$ \\
\hline $\mathbf{K}_{l h}$ & $1000-1$ & 0000 & 00000 & $\begin{array}{llll}-1 & 0 & 0 & 1\end{array}$ \\
\hline $\mathbf{K}_{r h}$ & $\begin{array}{llll}10 & 0 & 1\end{array}$ & $\begin{array}{lllll}0 & 0 & 0 & 0\end{array}$ & $\begin{array}{llll}0 & 0 & 0 & 0\end{array}$ & $\begin{array}{llll}1 & 0 & 0 & 1\end{array}$ \\
\hline
\end{tabular}
right helix $(r h)$. Table I presents their Kennaugh matrix form.

TABLE I: Kennaugh Matrices for Elementary Targets

The similarity measure defined in [18] corresponding to an elementary scatterer $\mathbf{K}_{i}$ is given as:

$$
f_{i}=1-G D\left(\mathbf{K}, \mathbf{K}_{i}\right)
$$

where $G D$ denotes the geodesic distance on the unit sphere in the Kennaugh matrix space [19]:

$$
G D\left(\mathbf{K}_{1}, \mathbf{K}_{2}\right)=\frac{2}{\pi} \cos ^{-1} \frac{\operatorname{Tr}\left(\mathbf{K}_{1}^{T} \mathbf{K}_{2}\right)}{\sqrt{\operatorname{Tr}\left(\mathbf{K}_{1}^{T} \mathbf{K}_{1}\right)} \sqrt{\operatorname{Tr}\left(\mathbf{K}_{2}{ }^{T} \mathbf{K}_{2}\right)}}
$$

where $\operatorname{Tr}$ is the trace operator. In this work, since our concern is primarily to extract built-up areas, we are only interested in three elementary scattering models: dihedral, narrow dihedral and helix.

The flowchart of the two approaches for the extraction of a built-up area map using PolSAR data is shown in Fig. 1. We discuss the steps in the following.

1) Input Scattering Models: The algorithm considers the elementary scattering models given in Table I along with the observed Kennaugh matrix $\mathbf{K}$ as input. Except for the helix, the rest of them are symmetric scatterers i.e., they have an axis of symmetry in the plane perpendicular to radar LOS.

2) Desying to Maximal Similarity: Very often, it is possible that in built-up areas the symmetric scatterers are placed obliquely to the radar LOS. Hence, in this situation, the target is deoriented using Huynen's desying operation:

$$
\begin{aligned}
& \mathbf{K}(\theta)=\mathbf{R}(\theta) \mathbf{K R}(\theta)^{T} ; \quad-\frac{\pi}{8} \leq \theta \leq \frac{\pi}{8} \\
& \mathbf{R}(\theta)=\left[\begin{array}{cccc}
1 & 0 & 0 & 0 \\
0 & \cos 2 \theta & -\sin 2 \theta & 0 \\
0 & \sin 2 \theta & \cos 2 \theta & 0 \\
0 & 0 & 0 & 1
\end{array}\right]
\end{aligned}
$$

This step takes into account the orientation of the observed target by determining which symmetric scatterer it closely corresponds to. This process leads to the determination of $\mathbf{K}_{m s}$, the $\mathbf{K}(\theta)$ for some $\theta=\theta_{m s}$ for which the similarity of the target with a symmetric scattering model is maximized, i.e., the geodesic distance is minimized:

$$
G D\left(\mathbf{K}\left(\theta_{m s}\right), \mathbf{K}_{j}\right)=\min _{\theta, i} G D\left(\mathbf{K}(\theta), \mathbf{K}_{i}\right)
$$

3) Scattering Similarity: The similarity of a target $\mathbf{K}_{m s}$ with the elementary scattering models is evaluated using the similarity measure, $f_{i}$, where $i$ denotes the subscript for each elementary scattering models. The vector $f=$ $\left[f_{d}, f_{n d}, \ldots, f_{l h}, f_{r h}\right]$ denotes the scattering similarities corresponding to each elementary target.

Once the scattering similarities for each elementary scattering models are obtained, two methods can be implemented to extract a built-up area map. Method I uses only the order of dominance of the similarities obtained in the last step (Step 3 above). This method needs no thresholding, whereas, Method II first proposes a radar built-up index which is then suitably thresholded by the Otsu method to map the built-up area.

\section{A. Method I}

1) Dominance Sorter: The scattering similarities are sorted in descending order. Vector $D=\left[f_{i_{1}}, f_{i_{2}}, \ldots, f_{i_{N}}\right]$ represents the dominance order of the scattering mechanisms, i.e., $f_{i_{1}} \geq f_{i_{2}} \geq \cdots \geq f_{i_{N}}$, where $f_{i_{j}}$ denotes the scattering similarity to the $j$-th dominant elementary scattering model and $N$ is the total number of elementary scattering models.

2) Built-up Condition: The first three dominant similarities $\overline{\left(f_{i_{1}}, f_{i_{2}} \text { and } f_{i_{3}}\right)}$ are individually matched with the elements from the set $\left\{f_{d}, f_{n d}, f_{l h}, f_{r h}\right\}$ i.e. the elementary 


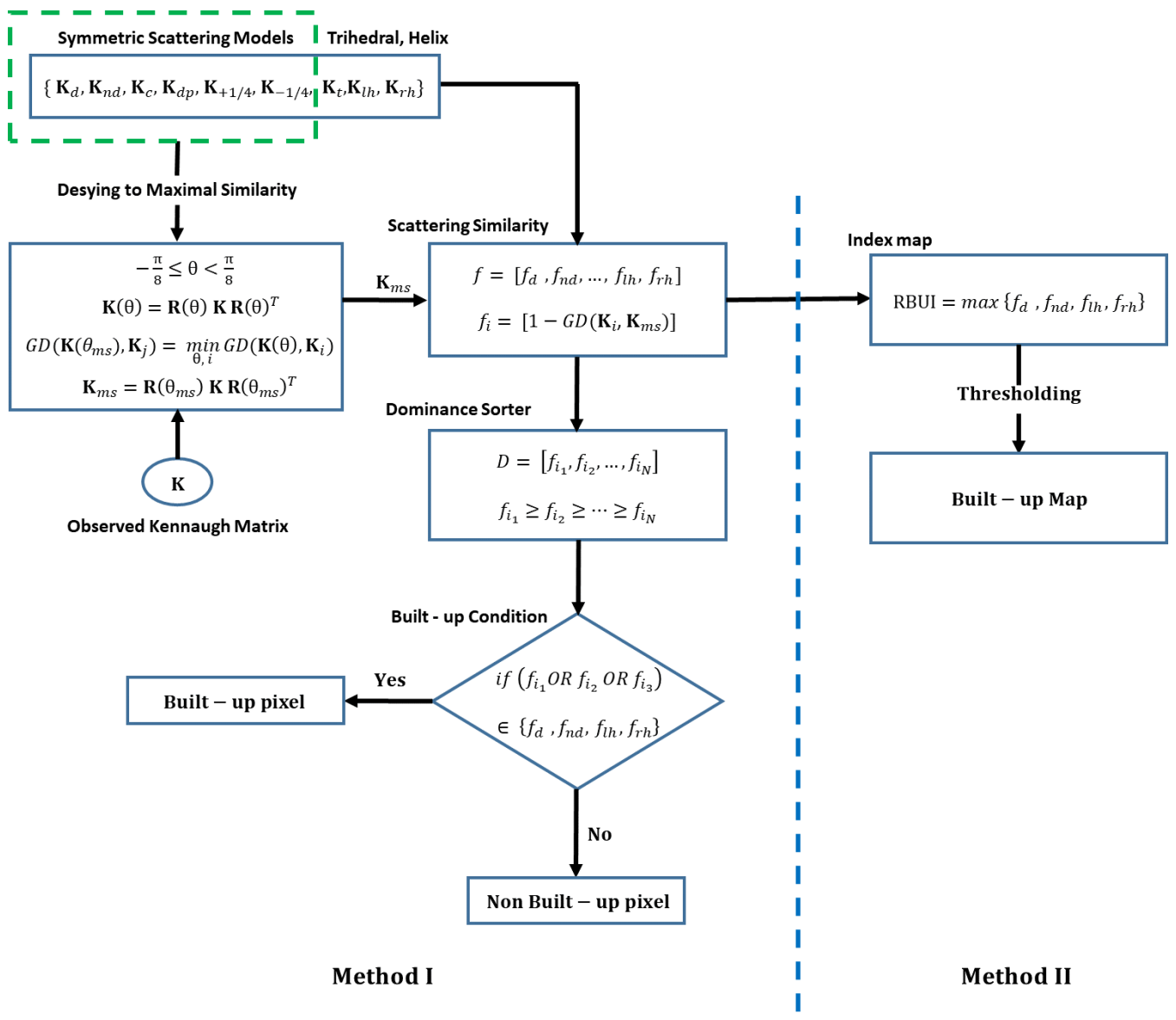

Fig. 1: Flowchart showing two methods for extraction of targets in the built-up area: Method I (threshold-free) and Method II (using thresholding)

scattering models corresponding to target in the built-up area, viz., dihedral, narrow dihedral and helix. If a match is found for atleast one of the $f_{i_{1}}, f_{i_{2}}$ and $f_{i_{3}}$, then the pixel is said to be of built-up type.

\section{B. Method II}

1) Index Map: Combining scattering similarities, a novel radar built-up index (RBUI) is proposed, defined as:

$$
\mathrm{RBUI}=\max \left\{f_{d}, f_{n d}, f_{l h}, f_{r h}\right\} ; \quad 0 \leq \mathrm{RBUI} \leq 1 .
$$

The higher the value of RBUI, the higher is the evidence of the pixel being of built-up type.

2) Thresholding: The RBUI is thresholded using the Otsu method [20] to form a binary ( 0 or 1 ) built-up area map.

In the next section, both the methods are quantitatively as well as qualitatively compared using PolSAR images and reference maps. These reference maps are generated by visual interpretation and fusion of geospatial data from multiple free sources.

\section{RESULTS AND DISCUSSION}

The results are shown using a RADARSAT-2 PolSAR image over San-Francisco, USA and an ALOS-2 L-band PolSAR image over Kyoto, Japan. The incidence angle varies from $28.0^{\circ}$ to $29.8^{\circ}$ from near to far range for the RADARSAT-2 image, and the scene-centered incidence angle is $30.9^{\circ}$ for the ALOS-2 image. The San-Francisco image ( $762 \times 978$ pixels) is multilooked by a factor of 2 in range and 4 in azimuth effectively providing a ground range pixel resolution of $20 \mathrm{~m}$ while the Kyoto image $(1616 \times 2826$ pixels $)$ is multilooked by a factor of 3 in range and 5 in azimuth providing a ground range pixel resolution of $15.7 \mathrm{~m}$.

Figs. 3(a) and 5(a) show the dominance of type scatterings prevalent in built-up areas. The pixels for which the similarity of $\mathbf{K}_{m s}$ among all the scattering models is highest for a built-up type scattering model, i.e., either the dihedral, narrow dihedral or the helices, are colored in red. Similarly, pixels for which built-up type scattering is second dominant whereas the first dominant is of non-built-up type are shown in green. Blue pixels indicate the third dominant scattering, and the remaining pixels are in black. It may be noted that only the first three dominant scattering mechanisms are compared with the builtup type models. Following this, a majority of the built-up areas are successfully captured using Method I; this can be seen in the built-up area maps presented in Figs. 3(b) and 5(b).

Table II shows the contribution of different built-up area scattering dominance types to built-up producer accuracy (\%) of Method I. It can be seen that most of the built-up area pixels have a built-up type scattering as the dominant scattering 
mechanism. The more difficult built-up area pixels which are severely oriented or situated on undulating terrain have builtup type scattering as secondary dominant mechanisms. This was why it is necessary to go beyond the most dominant scattering mechanism to detect more of the built-up area.

TABLE II: Contribution of different built-up area scattering dominance types to built-up area producer accuracy (\%) of Method I

\begin{tabular}{lccc}
\hline & I & II & III \\
\hline San-Francisco (RS-2) & 45 & 15 & 7 \\
\hline Kyoto (ALOS-2) & 50 & 13 & 5 \\
\hline
\end{tabular}

The radar built-up index (RBUI) maps for San Francisco, and Kyoto are shown in Figs. 4(a) and 6(a) respectively. The majority of built-up structures show a value greater than 0.5 , while water bodies and bare surface have values ranging between 0 and 0.1 . The areas with vegetation fall on the lower side of 0.5 . Hence, from these observations, 0.5 can be considered as a threshold to obtain the built-up area maps. However, a more systematic thresholding algorithm was employed in this study. The binary maps showing built-up areas in Figs. 4(b) and 6(b) were obtained by applying the Otsu thresholding technique [20].

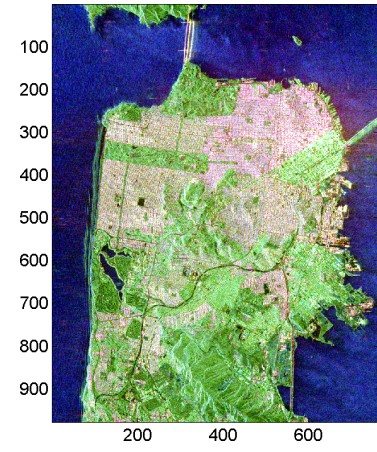

(a) Pauli RGB for SF

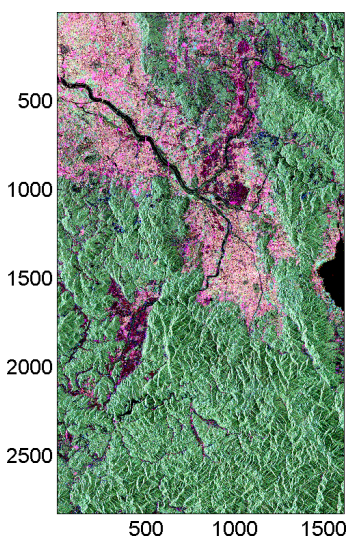

(c) Pauli RGB for KY

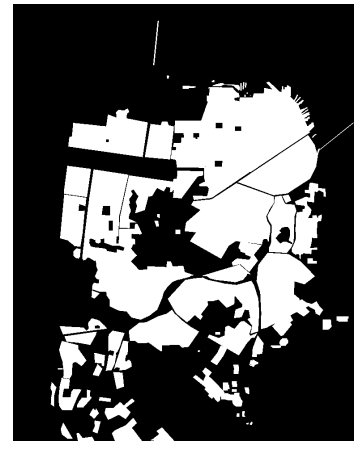

(b) Reference map for SF

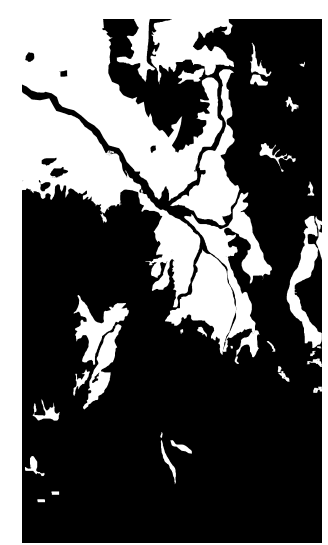

(d) Reference map for KY
Fig. 2: Pauli RGB and reference maps for built-up area within multi-looked RADARSAT-2 C-band image over San Francisco, USA and ALOS-2 L-band image over Kyoto, Japan.

Tables III and IV provide a quantitative assessment of the effectiveness of Method I and Method II for built-up area

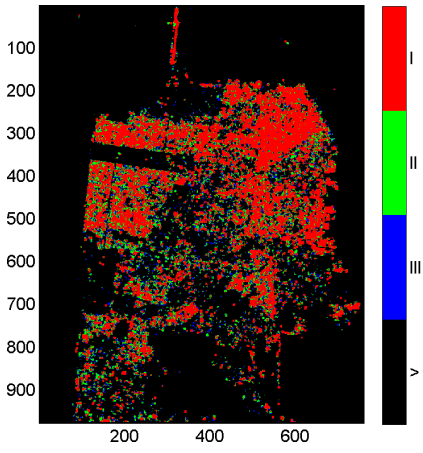

(a) Built-Up Area Scattering Dominance

Fig. 3: Built-Up Area Maps for multi-looked RADARSAT-2 C-band image over San Francisco, USA.

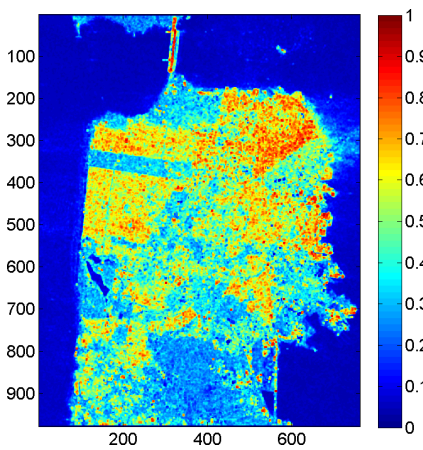

(a) Built-Up Index

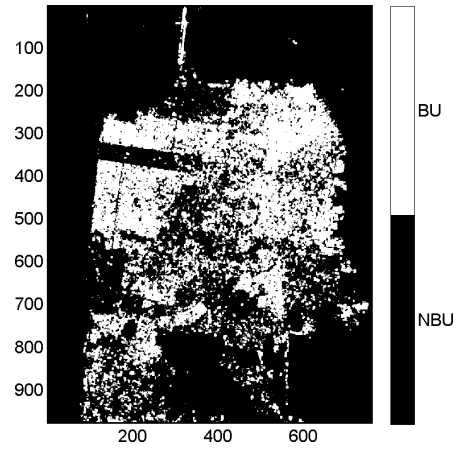

(b) Built-Up Area Map (Method I)
Fig. 4: Built-Up Area Maps for multi-looked RADARSAT-2 C-band image over San Francisco, USA.

extraction, respectively. The reference maps corresponding to the PolSAR scene of San Francisco and Kyoto are shown in Figs. 2(b) and 2(d). The performance of the two methods is assessed by:

- $P_{1}$ and $P_{2}$, the producer accuracy for built-up type (BU) and non-built-up type pixels (NBU) respectively.

- $O A$, the overall accuracy achieved with the method.

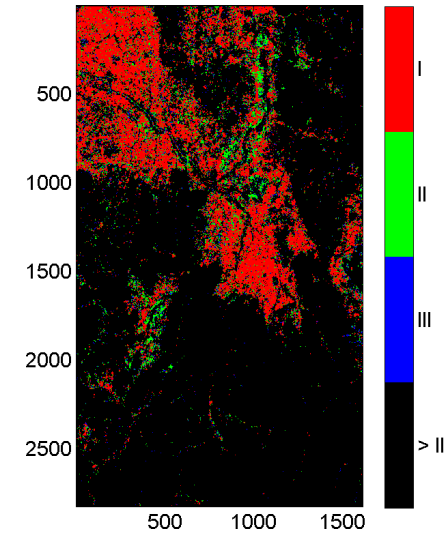

(a) Built-Up Area Scattering Dominance

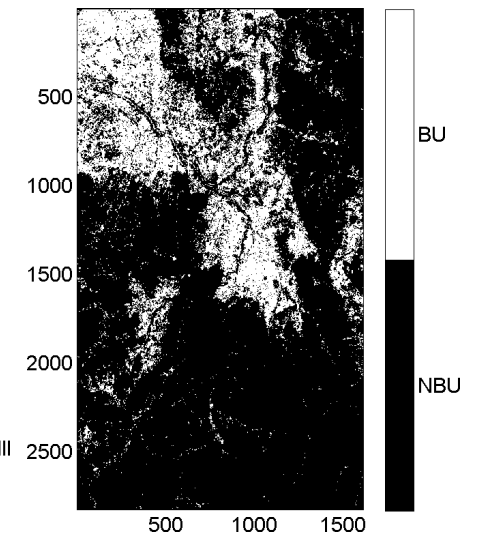

(b) Built-Up Area Map (Method I)
Fig. 5: Built-Up Area Maps for multi-looked ALOS-2 L-band image over Kyoto, Japan. 


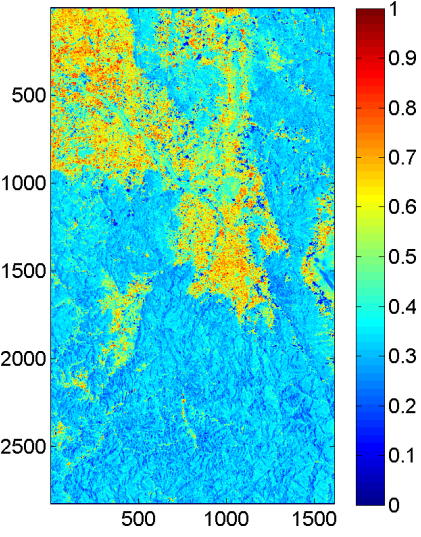

(a) Built-Up Index

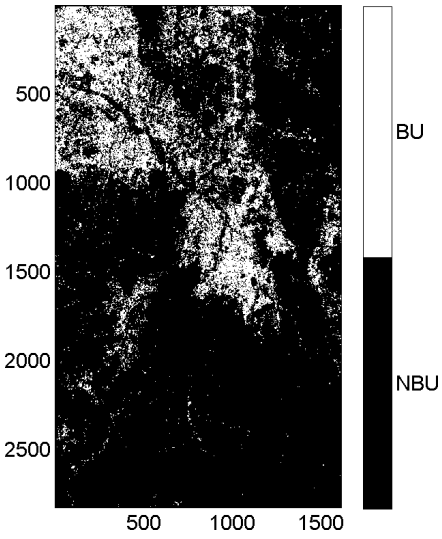

(b) Built-Up Area Map (Method II)
Fig. 6: Built-Up Area Maps for multi-looked ALOS-2 L-band image over Kyoto, Japan.

TABLE III: Performance Metrics in \%: Method I

\begin{tabular}{lccc}
\hline & $P_{1}$ & $P_{2}$ & $O A$ \\
\hline San-Francisco (RS-2) & 67 & 92 & 84 \\
\hline Kyoto (ALOS-2) & 68 & 95 & 88 \\
\hline
\end{tabular}

TABLE IV: Performance Metrics in \%: Method II

\begin{tabular}{lccc}
\hline & $P_{1}$ & $P_{2}$ & $O A$ \\
\hline San-Francisco (RS-2) & 69 & 92 & 85 \\
\hline Kyoto (ALOS-2) & 47 & 98 & 84 \\
\hline
\end{tabular}

From Tables III and IV, it is observed that Method I is slightly more robust than Method II. In particular, for the ALOS-2 scene, the former detects actual built-up area pixels in a more accurate way $\left(P_{1}=68 \%>47 \%\right)$, although both methods are comparable with respect to $P_{2}$ and $O A$.

The poor performance of Method II with respect to $P_{1}$ is due to the low dynamical range of RBUI over ALOS-2 scene as seen in Fig. 6(a) in comparison to Fig. 4(a) for the RADARSAT-2 scene. This results in a lack of clear decision boundary for the built-up area and non-built-up area pixels in the image for the ALOS-2 scene by the Otsu method. Method I is a categorical approach, hence devoid of such a difficulty. Instead, the RBUI can be utilized for situations that may require a continuous range built-up index, for instance, to map built-up area density.

\section{CONCLUSION}

In this work, two simple methodologies along with a new radar built-up index (RBUI) have been proposed for built-up area extraction from PolSAR imagery.

Method I demonstrates that the first three scattering mechanisms are sufficient for the detection of built-up area, whereas Method II shows the effectiveness of the proposed RBUI in differentiating the built-up area from bare regions.

Both methods achieve overall accuracy of around 80-90\% and exploit a notion of similarity to elementary scattering models, but they are different in the sense that they are categorical and quantitative, respectively.

It is worth noting, finally, that in this work the built-up area maps are a sort of "byproduct" of more useful maps, such as built-up area scattering dominance and RBUI values. In the future, besides exploiting this information more extensively, we plan to extend the methodology to bistatic radar datasets.

\section{REFERENCES}

[1] P. Gamba, "Human Settlements: A global challenge for EO data processing and interpretation," Proc. IEEE, vol. 101, no. 3, pp. 570-581, Mar. 2013.

[2] P. Gamba and M. Herold, Global mapping of human settlement: experiences, datasets, and prospects. CRC Press, 2009.

[3] F. M. Henderson and Z.-G. Xia, "SAR applications in human settlement detection, population estimation and urban land use pattern analysis: a status report," IEEE Trans. Geosci. Remote Sens., vol. 35, no. 1, pp. 79-85, Jan. 1997.

[4] P. Gamba and G. Lisini, "Fast and efficient urban extent extraction using ASAR wide swath mode data," IEEE J. Sel. Topics Appl. Earth Observ. Remote Sens., vol. 6, no. 5, pp. 2184-2195, Oct. 2013.

[5] G. Lisini, A. Salentinig, P. Du, and P. Gamba, "SAR-based urban extents extraction: From ENVISAT to Sentinel-1," IEEE J. Sel. Topics Appl. Earth Observ. Remote Sens., vol. 11, no. 8, pp. 2683-2691, Aug. 2018.

[6] M. Kajimoto and J. Susaki, "Urban-area extraction from polarimetric SAR images using polarization orientation angle," IEEE Geosci. Remote Sens. Lett., vol. 10, no. 2, pp. 337-341, Mar. 2013.

[7] B. Azmedroub, M. Ouarzeddine, and B. Souissi, "Extraction of urban areas from polarimetric SAR imagery," IEEE J. Sel. Topics Appl. Earth Observ. Remote Sens., vol. 9, no. 6, pp. 2583-2591, Jun. 2016.

[8] D. Duan and Y. Wang, "An improved algorithm to delineate urban targets with model-based decomposition of PolSAR data," Remote Sens., vol. 9, no. 10, p. 1037, Oct. 2017.

[9] D. Xiang, T. Tang, Y. Ban, and Y. Su, "Man-made target detection from polarimetric SAR data via nonstationarity and asymmetry," IEEE J. Sel. Topics Appl. Earth Observ. Remote Sens., vol. 9, no. 4, pp. 1459-1469, Feb. 2016.

[10] D. Xiang, T. Tang, C. Hu, Q. Fan, and Y. Su, "Built-up area extraction from PolSAR imagery with model-based decomposition and polarimetric coherence," Remote Sensing, vol. 8, no. 8, p. 685, Aug. 2016.

[11] W. Wu, H. Guo, and X. Li, "Urban area man-made target detection for PolSAR data based on a nonzero-mean statistical model," IEEE Geosci. Remote Sens. Lett., vol. 11, no. 10, pp. 1782-1786, Oct. 2014.

[12] S. De, L. Bruzzone, A. Bhattacharya, F. Bovolo, and S. Chaudhuri, "A novel technique based on deep learning and a synthetic target database for classification of urban areas in PolSAR data," IEEE J. Sel. Topics Appl. Earth Observ. Remote Sens., vol. 11, no. 1, pp. 154-170, Jan. 2018.

[13] R. Guinvarc'h and L. Thirion-Lefevre, "Cross-Polarization amplitudes of obliquely orientated buildings with application to urban areas," IEEE Geosci. Remote Sens. Lett., vol. 14, no. 11, pp. 1913-1917, Nov. 2017.

[14] L. Thirion-Lefevre and R. Guinvarc'h, "The double brewster angle effect," Comptes Rendus Physique, vol. 19, no. 1-2, pp. 43-53, 2018.

[15] D. K. Atwood and L. Thirion-Lefevre, "Polarimetric phase and implications for urban classification," IEEE Trans. Geosci. Remote Sens., vol. 56, no. 3, pp. 1278-1289, Mar. 2018.

[16] D. Ratha, A. Bhattacharya, and A. C. Frery, "A scattering power factorization framework using a geodesic distance in radar polarimetry," in Proc. 2018 IEEE Int. Geosci. Remote Sens. Symp. (IGARSS), Jul. 2018, pp. 6075-6078.

[17] J.-S. Lee and E. Pottier, Polarimetric radar imaging: from basics to applications. CRC press, 2009.

[18] D. Ratha, A. Bhattacharya, and A. C. Frery, "Unsupervised classification of PolSAR data using a scattering similarity measure derived from a geodesic distance," IEEE Geosci. Remote Sens. Lett., vol. 15, no. 1, pp. 151-155, Jan. 2018.

[19] D. Ratha, S. De, T. Celik, and A. Bhattacharya, "Change detection in polarimetric SAR images using a geodesic distance between scattering mechanisms," IEEE Geosci. Remote Sens. Lett., vol. 14, no. 7, pp. 10661070, Jul. 2017.

[20] N. Otsu, "A threshold selection method from gray-level histograms," IEEE Trans. Syst., Man, Cybern., vol. 9, no. 1, pp. 62-66, Jan. 1979. 